\title{
Physical Performance and Risk of Postoperative Delirium in Older Adults Undergoing Aortic Valve Replacement
}

This article was published in the following Dove Press journal:

Clinical Interventions in Aging

\author{
Aarti Rao (1) \\ Sandra M Shi $\mathbb{D}^{2,3}$ \\ Jonathan Afilalo $\mathbb{1 D}^{4}$ \\ Jeffrey J Popma ${ }^{5}$ \\ Kamal R Khabbaz ${ }^{6}$ \\ Roger J Laham ${ }^{5}$ \\ Kimberly Guibone 5 \\ Edward R Marcantonio (D) ${ }^{2,7}, *$ \\ Dae Hyun Kim (D) ${ }^{2,3, *}$
}

\begin{abstract}
'Icahn School of Medicine at Mount Sinai, New York, NY, USA; ${ }^{2}$ Division of Gerontology, Department of Medicine, Beth Israel Deaconess Medical Center, Boston, MA, USA; ${ }^{3}$ Hinda and Arthur Marcus Institute for Aging Research, Hebrew SeniorLife, Boston, MA, USA; ${ }^{4}$ Division of Cardiology and Centre for Clinical Epidemiology, Jewish General Hospital, McGill University, Montreal, Quebec, Canada; ${ }^{5}$ Division of Cardiology, Department of Medicine, Beth Israel Deaconess Medical Center, Boston, MA, USA; ${ }^{6}$ Division of Cardiac Surgery, Department of Surgery, Beth Israel Deaconess Medical Center, Boston, MA, USA; ${ }^{7}$ Division of General Medicine, Department of Medicine, Beth Israel Deaconess Medical Center, Boston, MA, USA
\end{abstract}

*These authors contributed equally to this work

Correspondence: Dae Hyun Kim Hinda and Arthur Marcus Institute for Aging Research, Hebrew SeniorLife, 1200 Centre Street, Boston, MA 0213I, USA

Tel + | 6|7-97|-8320

Fax + | 617-97|-5339

Email daehyunkim@hsl.harvard.edu
Background: Delirium is a major risk factor for poor recovery after surgical aortic valve replacement (SAVR) and transcatheter aortic valve replacement (TAVR). It is unclear whether preoperative physical performance tests improve delirium prediction.

Objective: To examine whether physical performance tests can predict delirium after SAVR and TAVR, and adapt an existing delirium prediction rule for cardiac surgery, which includes Mini-Mental State Examination (MMSE), depression, prior stroke, and albumin level.

Design: Prospective cohort, 2014-2017.

Setting: Single academic center.

Subjects: A total of 187 patients undergoing SAVR $(n=77)$ or TAVR $(n=110)$.

Methods: The Short Physical Performance Battery (SPPB) score was calculated based on gait speed, balance, and chair stands (range: 0-12 points, lower scores indicate poor performance). Delirium was assessed using the Confusion Assessment Method. We fitted logistic regression to predict delirium using SPPB components and risk factors of delirium.

Results: Delirium occurred in $35.8 \%$ (50.7\% in SAVR and $25.5 \%$ in TAVR). The risk of delirium increased for lower SPPB scores: 10-12 (28.2\%), 7-9 (34.5\%), 4-6 (37.5\%) and 0-3 (44.1\%) (p-for-trend=0.001). A model that included gait speed $<0.46$ meter/second (OR, 2.7; 95\% CI, 1.2-6.4), chair stands time $\geq 11.2$ seconds (OR, 3.5; 95\% CI, 1.0-12.4), MMSE $<24$ points (OR, 2.9; 95\% CI, 1.3-6.4), isolated SAVR (OR, 5.4; 95\% CI, 2.1-13.8), and SAVR and coronary artery bypass grafting $(\mathrm{OR}, 15.8 ; 95 \% \mathrm{CI}, 5.5-45.7)$ predicted delirium better than the existing prediction rule (C statistics: 0.71 vs $0.61 ; \mathrm{p}=0.035$ ).

Conclusion: Assessing physical performance, in addition to cognitive function, can help identify high-risk patients for delirium after SAVR and TAVR.

Keywords: delirium, physical performance, aortic valve replacement, prediction

\section{Introduction}

Postoperative delirium is a life-threatening complication after aortic valve replacement - surgical (SAVR) and transcatheter (TAVR) procedures - characterized by an acute onset of fluctuating cognitive dysfunction. ${ }^{1-3}$ It most commonly affects older patients as a result of predisposing risk factors (eg, cognitive impairment, severe illness) and precipitating stressors (eg, surgery, hospitalization). ${ }^{3}$ Contrary to earlier views of delirium as a transient change in mental status with full recovery, recent evidence suggests that delirium after cardiac surgery can result in persistent cognitive impairment, functional decline, and mortality. ${ }^{2,4,5}$ 
Patients undergoing SAVR appear to be at a higher risk of postoperative delirium compared to those undergoing other cardiac surgeries. ${ }^{6}$ While TAVR is a less invasive treatment option for high-risk patients, ${ }^{7}$ TAVR patients seem to develop more severe delirium with worse functional decline compared to SAVR patients, likely as a result of their advanced age, multimorbidity, and lower physiologic reserve. ${ }^{1,8}$ Balancing the risks of postoperative delirium with the need for aortic valve replacement to prevent the fatal progression of symptomatic aortic stenosis ${ }^{9}$ can be challenging for physicians. Given the lack of effective treatments for delirium, prevention currently remains the most important strategy for mitigating the potential long-term complications of postoperative delirium. ${ }^{10}$

Delirium prediction rules enable healthcare providers to identify high-risk patients who are most likely to benefit from screening and prevention efforts. In 2009, Rudolph et al developed and validated a delirium prediction rule (hereafter referred to as the Rudolph model) after cardiac surgery ${ }^{11}$ based on established risk factors for delirium, namely, cognitive impairment, depression, prior stroke or transient ischemic attack, and abnormal serum albumin. ${ }^{12-14}$ As this model was derived from patients (mean age: 74.7 years) primarily undergoing urgent coronary artery bypass grafting surgery (CABG), it is unclear whether the model can predict delirium in elective aortic valve replacement patients, particularly TAVR patients, who are older, frailer, and with a higher burden of delirium risk factors than CABG patients. ${ }^{7}$ Furthermore, due to the urgent nature of the surgery, the Rudolph model could not incorporate physical performance measures. Recent evidence indicates that poor physical performance may predispose older adults to delirium across various surgeries, including cardiac surgery. ${ }^{15}$ As such, it may be beneficial to adapt the Rudolph model for SAVR and TAVR patients by including physical performance measures.

This study aimed to evaluate how well the Rudolph model predicts delirium in older patients undergoing aortic valve replacement. We then examined whether physical performance tests can improve delirium prediction when added to the Rudolph model.

\section{Methods}

\section{Study Design and Participants}

This single-center prospective cohort study was conducted to evaluate functional outcomes after aortic valve replacement. Details of study design are described elsewhere. ${ }^{1,2}$ Between
February 2014 and March 2016, we screened 446 patients who were 70 years or older and undergoing evaluation for SAVR or TAVR. We excluded 96 patients based on the following criteria (not mutually exclusive): did not have severe aortic stenosis $(\mathrm{n}=35)$, had a concurrent surgery involving the aorta or other heart valves $(\mathrm{n}=28)$, were medically unstable for study assessment (eg, active myocardial ischemia or unstable vital signs) $(\mathrm{n}=9)$, had severe neuropsychological impairment (eg, Mini-Mental State Examination [MMSE] score $<15$ points or active psychosis) $(\mathrm{n}=4)$, or were non-English speaking $(\mathrm{n}=25)$. We further excluded 104 patients due to unavailability of the research team $(n=60)$, patient refusal $(n=39)$, or other reasons (eg, those who did not undergo the planned procedure, were enrolled in an interventional study, or withdrew consent after baseline assessment) ( $\mathrm{n}=5)$, leaving 246 patients for the study of functional outcomes. ${ }^{2}$

The standardized assessment of delirium (delirium substudy) began in October 2014, and included 187 consecutive patients who were enrolled between October 2014 and March 2016 (see a flow diagram in Supplementary Figure 1). Patients included in the delirium sub-study had a lower 30 day predicted risk of mortality than those enrolled prior to the sub-study ( $5.8 \%$ vs $4.2 \%$ ); however, other characteristics were similar (Supplementary Table 1). The Beth Israel Deaconess Medical Center Institutional Review Board approved this study. Study procedures were performed in accordance with the Declaration of Helsinki. All participants provided written informed consent.

\section{Baseline Measurements}

A research nurse or a trained research assistant assessed participants in the ambulatory testing center or hospital ward prior to the procedure and reviewed medical records to obtain the following information: demographic characteristics; Charlson index; the Society of Thoracic Surgeons Predicted Risk of Mortality; ${ }^{16}$ 5-item Geriatric Depression Scale; ${ }^{17}$ MMSE; and the Short Physical Performance Battery (SPPB) (described below). ${ }^{18}$

\section{The Rudolph Delirium Prediction Model}

The delirium risk score by the Rudolph model (range: $0-5$ points) was calculated based on the following scoring system: MMSE $\leq 23$ points received 2 points; MMSE 24-27 1 point, positive screening for depression (5-item Geriatric Depression Scale $\geq 2$ points), prior stroke or transient ischemic attack, and abnormal serum albumin level $(<3.5$ or $>5.2 \mathrm{~g} / \mathrm{dL})$ received 1 point each. We 
stratified patients into the following risk categories: $0,1,2$, and $3-5$ points. ${ }^{11}$

\section{Short Physical Performance Battery}

The SPPB is a measure of lower extremity function that consists of gait speed from a timed 5-meter walk test, 5-chair stands, and a hierarchal standing balance test. ${ }^{18}$ To measure gait speed (meters/second), participants were asked to walk 5-meters at their usual pace (use of assisted device was allowed). For 5-chair stands test, participants were asked to stand up from a chair 5 times as quickly as possible with their arms folded across their chests. If they were unable to complete the task, 60 seconds were assigned (60 seconds were the maximum time we allowed for the task). To assess standing balance, participants were asked to stand in 3 progressively challenging positions for up to 10 -seconds each, only advancing after successful completion of the easier stance: side-by-side (when feet are together), semi-tandem (when one foot is partially in front of the other), and tandem positions (when one foot is completely in front of the other). ${ }^{18}$ Each task is scored 0 to 4 points based on the previously defined population quartile cutpoints, with 0 points for tasks individuals are unable to complete and 4 points for the highest level of performance. ${ }^{18}$ The scores for each task are summed for a total score ranging from $0-12$ points. Lower scores are associated with mortality and disability. ${ }^{19,20}$ We stratified patients into the following categories using the original cutpoints: ${ }^{19,20} 0-3,4-6,7-9$, and $10-12$ points.

\section{Assessment of Delirium and Delirium Severity}

From postoperative day 1 through the day before discharge, a study geriatrician or a trained research assistant interviewed patients, nursing staff, and family members (if available), and administered standardized assessments of attention (digit span, day-of-the-week backwards, and month-of-the-year backwards), MMSE, and Delirium Symptom Interview. ${ }^{21}$ The in person assessments were completed in 15-25 minutes, during a consistent time of day. Based on this information, delirium was diagnosed according to the Confusion Assessment Methods (CAM) algorithm: 1) acute onset or fluctuating course, 2) inattention, and 3) either disorganized thinking or an altered level of consciousness. ${ }^{22}$ For intubated patients, we used CAM for Intensive Care Unit. ${ }^{23}$ The CAM has a sensitivity of $94 \%$ and a specificity of $89 \%$ in detecting delirium. ${ }^{24}$ The inter-rater reliability for delirium diagnosis between the study geriatricians and research assistants assessed in 30 assessments conducted in 10 patients were high (agreement $95-100 \%$, Cohen's kappa 0.90-1.00). ${ }^{25}$ Delirium severity was assessed using a validated CAM-based severity scale, CAM-S, which ranges from 0 to 19 with high scores indicating greater severity. ${ }^{23}$ No severity score was assigned when CAM for Intensive Care Unit was used. For each individual, we assigned the maximum CAM-S score for the index hospitalization.

\section{Statistical Analysis}

As some patients were unable to complete physical performance testing, in order to minimize bias from missing data, we imputed missing data on gait speed $(n=25)$, SPPB balance score $(\mathrm{n}=19)$ using a multivariable imputation by chained equations ${ }^{26}$ based on preoperative clinical characteristics, complications, functional status, and mortality during the 12-month follow-up. Including variables collected in the postoperative follow-up period in the imputation model is important to maintain the strength of associations among these variables. ${ }^{27}$ Baseline characteristics of patients who developed delirium and those who did not were compared using two-sample $t$-test or chisquare test.

To evaluate the predictive performance of the Rudolph model, we estimated delirium risk for $0,1,2$, and $3-5$ points in the total population, and separately in procedural cohorts. We also estimated the risk of delirium for total SPPB score categories (0-3, 4-6, 7-9, 10-12 points) and individual component scores. We fitted logistic regression to estimate the odds ratio (OR) and $95 \%$ confidence interval (CI) of delirium associated with the risk categories according to the Rudolph model and SPPB, adjusting for procedure type. Discrimination was evaluated using C-statistics. In addition, we examined the independent association between individual SPPB components and delirium in the logistic model.

Because not all of the components of the Rudolph model and SPPB may be equally important for predicting delirium, we performed variable selection to minimize the Akaike Information Criterion. When there was a nonlinear association (or threshold effect) between a predictor and delirium, the variables were dichotomized at a cutpoint where the strength of association changed. The C-statistic of the final model was compared with that of Rudolph model using 1000 bootstrap resampling. We 
developed a revised risk scoring system by assigning integer points proportional to the regression coefficients.

As a secondary analysis, we examined whether the Rudolph model, SPPB score, and the new risk score from our adapted model were correlated with delirium severity by scatter plots and Spearman correlation coefficients. All analyses were performed with Stata Release 14 (StataCorp, College Station, Texas). A 2-sided p-value $<0.05$ was considered statistically significant.

\section{Results}

Of the 187 patients, delirium occurred in 67 (35.8\%) (39 SAVR patients and 28 TAVR patients) (Table 1). Patients who developed delirium had lower mean MMSE scores ( 25.0 vs 26.0 points), lower balance scores (2.3 vs 2.8 points), and were more likely to have undergone concomitant SAVR and CABG (31.3\% vs $8.3 \%)$. Other baseline characteristics did not differ between patients who did or did not develop delirium. The maximum CAM-S score was higher in those with delirium ( 8.9 vs 3.3 points).

\section{Evaluation of the Rudolph Delirium Prediction Model}

According to the Rudolph model risk categories, the incidence of delirium was $31.7 \%$ (13/41 patients) for 0 points, $33.9 \%$ (20/59 patients) for 1 point, $32.6 \%$ (15/46 patients) for 2 points, and $46.3 \%$ (19/41 patients) for 3-5 points (Supplementary Table 2). After adjusting for procedure type, higher Rudolph model risk categories were associated with delirium ( $p$-value for trend $=0.007$ ). The Rudolph model C-statistic was 0.61 (95\% CI, 0.50-0.70) in the total population, $0.60(0.49-0.71)$ in SAVR cohort, and $0.62(0.51-0.73)$ in TAVR cohort. The results for each procedure cohort were similar.

\section{Physical Performance and Risk of Delirium}

The risk of delirium increased for with lower SPPB scores: $28.2 \%$ (11/39 patients) for $10-12$ points, $34.5 \%$ (20/58 patients) for $7-9$ points, $37.5 \%$ (21/56 patients) for 4-6 points, and $44.1 \%$ (15/34 patients) for $0-3$ points (Table 2). Delirium risk rose with lower SPPB scores category ( $\mathrm{p}$-value for trend $=0.001$ ), after adjusting for procedure type. The results were similar in each procedure cohort (Supplementary Table 3). As scores of the individual SPPB components (balance, gait speed, and chair stand) decreased, risk of delirium increased (Table 2, model 1) after adjusting for procedure type. However, when all 3 components were included in the model, none of the components were associated with the risk of delirium (Table 2, model 2).

The C-statistic of total SPPB score after adjusting for procedure type was $0.66(95 \% \mathrm{CI}, 0.55-0.75)$ in the total population, $0.71(0.59-0.81)$ in SAVR, and $0.62(0.52--$ 0.73 ) in TAVR (Supplementary Table 3). Adding SPPB to the Rudolph model improved the $\mathrm{C}$-statistic in the total population ( 0.61 to 0.68 ; $\mathrm{p}$-value $=0.038)$ and SAVR cohort ( 0.60 to $0.75 ; \mathrm{p}$-value $=0.030$ ), but not in TAVR cohort ( 0.62 to 0.67 ; $p$-value $=0.305$ ).

\section{An Adapted Delirium Risk Scoring System}

When we fitted a multivariable logistic model that included 4 components of the Rudolph model, SPPB components, and procedure type, the final model that achieved the lowest Akaike Information Criterion included gait speed, chair stand time, MMSE score, and procedure type (Supplementary Table 4). This model showed a C-statistic of 0.71 (95\% CI, 0.61-0.80) in the total population, which was better than that of Rudolph model $(\mathrm{p}=0.035)$. The predicted risk of delirium from this model corresponded well to the observed risk of delirium in our population (Figure 1).

\section{Delirium Risk Scores and Delirium}

\section{Severity}

Both the Rudolph risk score (correlation=0.29) and SPPB total score (correlation $=-0.19$ ) were correlated with the maximum CAM-S score (Figure 2). The correlation was stronger for the adapted model risk score (correlation=0.40).

\section{Discussion}

In this prospective cohort study of older adults undergoing elective aortic valve replacement, we showed that poor physical performance, as measured using the SPPB, was associated with higher risk of postoperative delirium and its severity. Adding physical performance measures improved the predictive performance of a validated Rudolph delirium prediction model. ${ }^{11}$ We then adapted the Rudolph model for aortic valve replacement patients by developing a simple scoring system based on gait speed, chair stands, MMSE, and procedure type.

The Rudolph model is one of the few validated preoperative delirium prediction models for cardiac surgery. ${ }^{11}$ This model achieved a C-statistic of 0.75 in the original cohort of predominantly urgent CABG patients. However, 
Table I Characteristics of Study Population

\begin{tabular}{|c|c|c|c|c|}
\hline Characteristics & Total $(\mathbf{N}=187)$ & No Delirium $(\mathrm{N}=\mid 20)$ & Delirium ( $N=67)$ & P-value \\
\hline Age, years, mean \pm SD & $81.3 \pm 6.4$ & $81.5 \pm 6.8$ & $81.0 \pm 5.7$ & 0.579 \\
\hline Female, n (\%) & $90(48.1)$ & $58(48.3)$ & $32(47.8)$ & 0.940 \\
\hline White race, n (\%) & $180(96.3)$ & II $5(95.8)$ & $65(97.0)$ & 0.683 \\
\hline Charlson index (range: $0-33$ ), ${ }^{\mathrm{a}}$ mean $\pm \mathrm{SD}$ & $3.1 \pm 2.2$ & $3.2 \pm 2.3$ & $3.0 \pm 2.0$ & 0.534 \\
\hline STS-PROM, ${ }^{a} \%$, mean \pm SD & $4.2 \pm 2.5$ & $4.4 \pm 2.6$ & $3.9 \pm 2.2$ & 0.207 \\
\hline Gait speed, meters/second, mean \pm SD & $0.7 \pm 0.3$ & $0.7 \pm 0.3$ & $0.7 \pm 0.3$ & 0.646 \\
\hline Chair stands, seconds, mean \pm SD & $33.1 \pm 21.5$ & $32.6 \pm 21.6$ & $33.9 \pm 21.4$ & 0.709 \\
\hline \multicolumn{5}{|l|}{ Rudolph delirium prediction model } \\
\hline Total score (range: $0-5$ ), mean \pm SD & $1.5 \pm 1.2$ & $1.5 \pm 1.2$ & $1.6 \pm 1.2$ & 0.378 \\
\hline MMSE (range: $0-30),{ }^{b}$ mean \pm SD & $25.6 \pm 3.2$ & $26.0 \pm 3.1$ & $25.0 \pm 3.4$ & 0.046 \\
\hline Depression, n (\%) & $59(32.0)$ & $35(29.2)$ & $24(35.8)$ & 0.348 \\
\hline Stroke or TIA, n (\%) & $28(15.0)$ & $22(18.3)$ & $6(9.0)$ & 0.085 \\
\hline Abnormal albumin, $\mathrm{n}(\%)$ & $19(10.2)$ & $12(10.0)$ & $7(10.5)$ & 0.952 \\
\hline \multicolumn{5}{|l|}{$\mathrm{SPPB}^{\mathrm{b}}$} \\
\hline Total score (range: $0-12$ ), mean $\pm S D$ & $6.8 \pm 2.9$ & $7.0 \pm 2.9$ & $6.4 \pm 2.9$ & 0.199 \\
\hline Gait speed score (range: $0-4$ ), mean \pm SD & $2.5 \pm 1.2$ & $2.5 \pm 1.2$ & $2.5 \pm 1.2$ & 0.894 \\
\hline Chair stand score (range: $0-4$ ), mean \pm SD & $1.6 \pm 1.0$ & $1.7 \pm 1.0$ & $1.6 \pm 0.9$ & 0.449 \\
\hline Balance score (range: $0-4$ ), mean $\pm S D$ & $2.6 \pm 1.4$ & $2.8 \pm 1.3$ & $2.3 \pm 1.5$ & 0.040 \\
\hline Procedure type & & & & $<0.001$ \\
\hline SAVR isolated, $n(\%)$ & $46(24.6)$ & $28(23.3)$ & $18(26.9)$ & \\
\hline SAVR and CABG, n (\%) & $31(16.6)$ & $10(8.3)$ & $21(31.3)$ & \\
\hline TAVR, n (\%) & $110(58.8)$ & $82(68.3)$ & $28(4 I .8)$ & \\
\hline CAM-S maximum (range: $0-19$ ), ${ }^{\mathrm{a}}$ mean $\pm \mathrm{SD}$ & $5.3 \pm 3.4$ & $3.3 \pm 1.8$ & $8.9 \pm 2.6$ & $<0.001$ \\
\hline
\end{tabular}

Notes: ${ }^{a}$ Higher scores indicate higher mortality for Charlson index and STS-PROM and severe delirium for CAM-S. 'bower scores indicate lower performance. Abbreviations: CABG, coronary artery bypass grafting; CAM-S, Confusion Assessment Methods-Based Severity scale; MMSE, Mini-Mental Status Examination; SAVR, surgical aortic valve replacement; SD, standard deviation; SPPB, Short Physical Performance Battery; STS-PROM, Society of Thoracic Surgeons Predicted Risk of Mortality; TAVR, transcatheter aortic valve replacement; TIA, transient ischemic attack.

its lower C-statistic of 0.61 in our cohort suggests that predictors of delirium in older and frailer patients undergoing elective SAVR and TAVR may differ from those in urgent CABG patients. Low MMSE score was strongly associated with delirium in the Rudolph's cohort and in our cohort, but high Geriatric Depression Scale score, history of stroke, and abnormal albumin level were not predictive of delirium in our study. Because the pathophysiology of calcific aortic stenosis differs from that of atherosclerotic coronary artery disease, ${ }^{28}$ risk factors that are sequelae of long-term cerebrovascular disease, such as prior stroke and depressive symptoms, may be less predictive of delirium in elective aortic valve replacement patients.

Impairment in physical performance is highly prevalent in older adults undergoing SAVR and TAVR. ${ }^{2,29}$ Recent literature has increasingly demonstrated an association between physical performance measures or frailty and postoperative delirium. ${ }^{13,30}$ Studies of patients undergoing CABG and TAVR have found that low SPPB scores are associated with an increased risk of delirium (OR for 4-6 vs $10-12$ points: 8.26$).{ }^{31}$ Similarly, in non-cardiac patients, physical frailty was associated with delirium (OR for frail vs non-frail group ranged from 1.54 to 1.96$){ }^{32,33}$ Our study builds upon this previous research by showing that lower SPPB scores are associated with delirium risk (OR for $0-3$ vs 10-12 points: 6.9) and that adding SPPB to the Rudolph model significantly improved the predictive power of the Rudolph model in the total population (C-statistics 0.61 to $0.68)$. The improvement was notable in SAVR patients $(0.60$ to 0.75 ) and less so in TAVR patients (0.62 to 0.67 ). Furthermore, our adapted model was more strongly correlated with delirium severity than the Rudolph model.

Our findings shed light on a potential underlying mechanism of poor physical performance as a risk factor for postoperative delirium. The independent association between SPPB and delirium suggests that an evaluation of physical performance may provide an indirect assessment of brain vulnerabilities that are distinct from the memory pathways assessed by MMSE. ${ }^{34}$ Similarly, slower gait speed has been 
Table 2 Physical Performance and Risk of Delirium After Aortic Valve Replacement

\begin{tabular}{|c|c|c|c|c|}
\hline SPPB & $\mathbf{N}_{\text {At Risk }}$ & $\mathbf{N}_{\text {Delirium }}(\%)$ & Model I OR $(95 \% \mathrm{Cl})^{\mathrm{a}}$ & Model 2 OR $(95 \% \mathrm{Cl})^{\mathrm{b}}$ \\
\hline \multicolumn{5}{|l|}{ Total score } \\
\hline $10-12$ points & 39 & II (28.2) & Reference & NA \\
\hline 7-9 points & 58 & $20(34.5)$ & $2.8(1.1-7.5)$ & NA \\
\hline 4-6 points & 56 & $21(37.5)$ & $4.1(1.5-11.6)$ & NA \\
\hline \multirow[t]{2}{*}{$0-3$ points } & 34 & $15(44.1)$ & $6.9(2.1-22.5)$ & NA \\
\hline & & & $P_{\text {trend }}=0.001$ & NA \\
\hline \multicolumn{5}{|l|}{ Gait speed score } \\
\hline 4 points & 58 & $22(37.9)$ & Reference & Reference \\
\hline 3 points & 27 & $9(33.3)$ & $1.4(0.5-4.1)$ & $1.0(0.3-3.1)$ \\
\hline 2 points & 54 & $16(29.6)$ & $1.6(0.6-4.0)$ & $1.0(0.3-2.9)$ \\
\hline \multirow[t]{2}{*}{$0-1$ points } & 48 & $20(4 \mid .7)$ & $4.3(1.5-12.6)$ & $2.6(0.7-9.2)$ \\
\hline & & & $P_{\text {trend }}=0.012$ & $P_{\text {trend }}=0.207$ \\
\hline \multicolumn{5}{|l|}{ Chair stand score } \\
\hline 4 points & 18 & $4(22.2)$ & Reference & Reference \\
\hline 3 points & 18 & $8(44.4)$ & $4.2(0.9-18.6)$ & $3.8(0.8-17.6)$ \\
\hline 2 points & 30 & $10(33.3)$ & $3.4(0.8-13.7)$ & $3.3(0.8-14.1)$ \\
\hline \multirow[t]{2}{*}{$0-I$ points } & 121 & $45(37.2)$ & $5.6(1.6-19.9)$ & $3.8(0.9-16.4)$ \\
\hline & & & $P_{\text {trend }}=0.014$ & $P_{\text {trend }}=0.147$ \\
\hline \multicolumn{5}{|l|}{ Balance score } \\
\hline 4 points & 74 & $22(29.7)$ & Reference & Reference \\
\hline 3 points & 33 & II (33.3) & $1.7(0.6-4.2)$ & I.5 $(0.6-4.0)$ \\
\hline 2 points & 24 & $9(37.5)$ & $2.1(0.8-6.1)$ & I.5 (0.5-4.5) \\
\hline \multirow[t]{2}{*}{$0-1$ points } & 56 & $25(44.6)$ & $2.8(1.3-6.3)$ & I.5 (0.6-4.0) \\
\hline & & & $P_{\text {trend }}=0.010$ & $P_{\text {trend }}=0.365$ \\
\hline
\end{tabular}

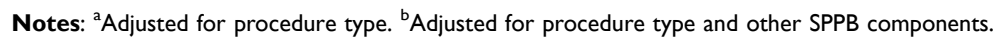

Abbreviations: $\mathrm{Cl}$, confidence interval; NA, not applicable; OR, odds ratio; SPPB, Short Physical Performance Battery.

associated with postoperative delirium after urological surgery. ${ }^{35}$ Previous work has demonstrated that regular exercise is significantly protective against incident delirium, potentially by improving cognitive function due to increases in gray and white matter volume in prefrontal and temporal cortices. Thus those able to engage in regular exercise may experience less neural decline. ${ }^{36}$ Alternatively, exercise may upregulate production of brain-derived neurotrophic factor, which is involved in neurogenesis and neuroprotection. ${ }^{37}$ Other evidence suggests that patients with impaired mobility often have white matter lesions, ${ }^{38}$ which in turn may predispose them to delirium in the face of surgical stressors. SPPB improves delirium prediction in cardiac surgery patients; however, these were done in cohorts that are generally lower risk, compared to the frailer TAVR cohort. ${ }^{31}$ Our results also support an intriguing hypothesis that prehabilitation and preoperative optimization of physical performance through exercise and nutritional intervention may be effective in preventing delirium. ${ }^{39}$ This hypothesis should be investigated in future research.
Based on our findings, it may be beneficial to include an assessment of physical performance into pre-operative evaluation of elective aortic valve replacement patients, in addition to a cognitive assessment. To this end, we adapted the Rudolph model, which accounted for gait speed, chair stands, MMSE, and procedure type, to estimate the risk of delirium after aortic valve replacement. While C-statistics were not significantly improved in the TAVR cohort due to being underpowered, there was a statistically significant improvement in discrimination in the overall population and SAVR cohort. In the United States, measurement of gait speed is mandatory before TAVR. Chair stands test is practical because it can be completed within 60 seconds with a chair and a stopwatch in a clinic room. Those who are unable to complete a task are assigned 0 points, thereby allowing assessment of physical performance in a full spectrum. From the perspective of pre-operative prediction, our model can be useful for deciding about the type of aortic valve replacement procedure and identifying target patients for delirium prevention. Candidate 


\begin{tabular}{|l|c|}
\hline \multicolumn{1}{|c|}{ Risk Score } & Points \\
\hline Gait speed $<0.46$ meters/second & 1 \\
\hline 5 Repeated chair stands $\geq 11.2$ seconds & 1 \\
\hline MMSE score < 24 points & 1 \\
\hline Procedure type: isolated SAVR & 2 \\
\hline Procedure type: SAVR and CABG & 3 \\
\hline Total Score & \\
\hline
\end{tabular}

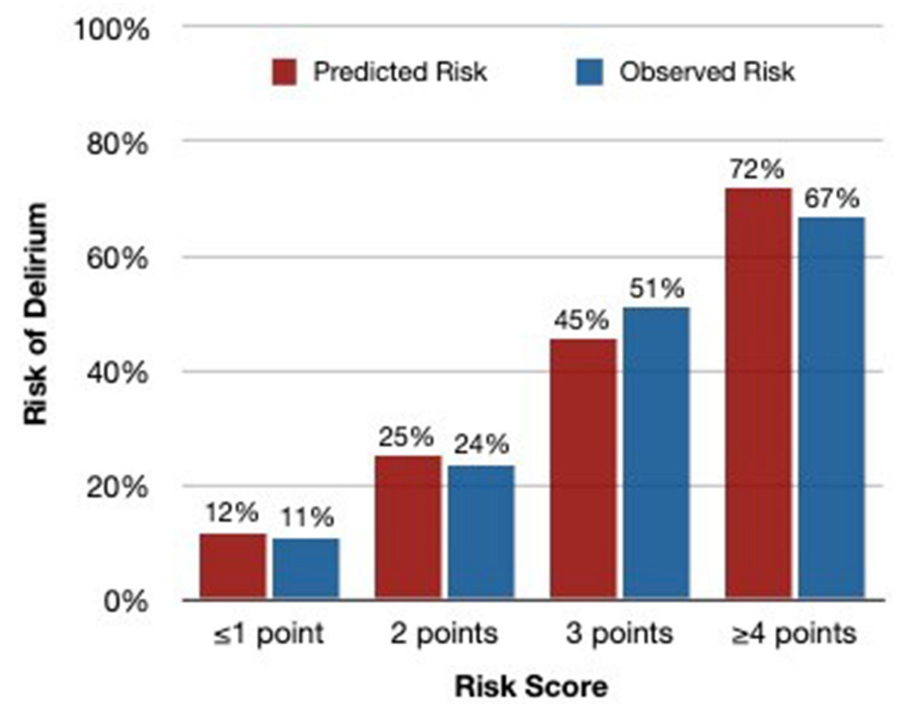

Figure I An adapted delirium risk scoring system for aortic valve replacement.

Abbreviations: CABG, coronary artery bypass grafting; MMSE, Mini-Mental Status Examination; SAVR, surgical aortic valve replacement.
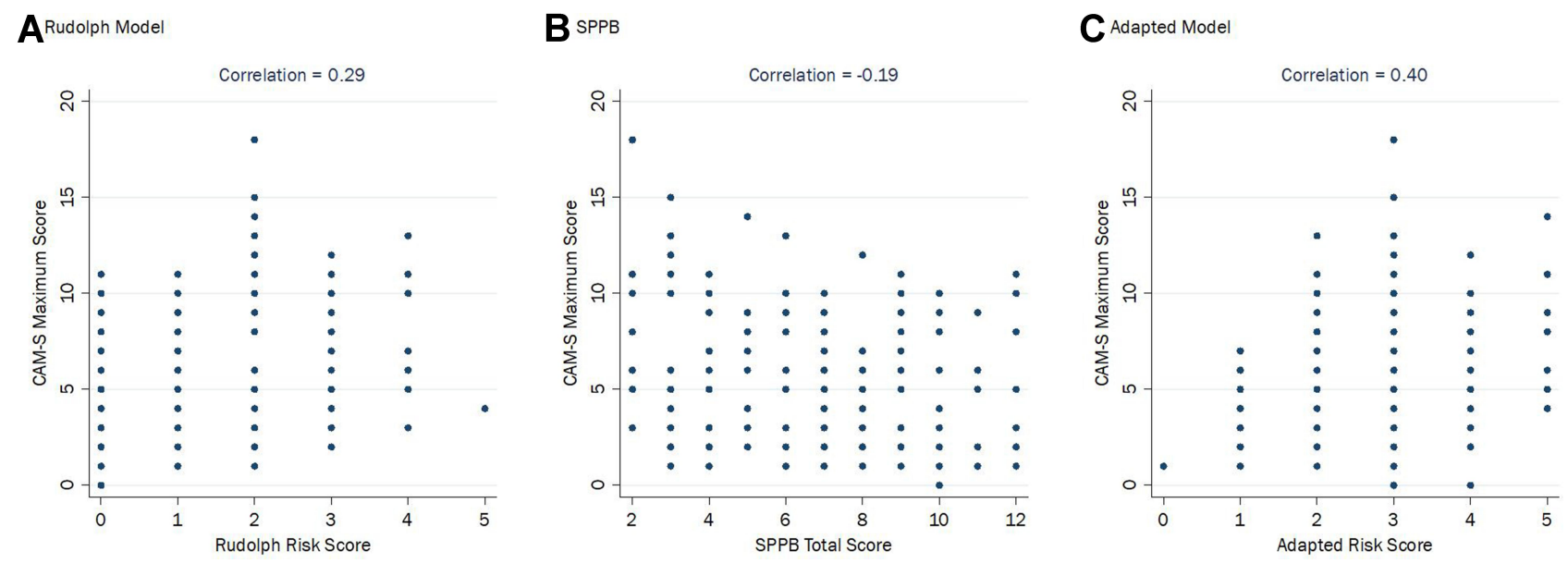

Figure 2 Delirium prediction risk scores and delirium severity. (A) Spearman correlation coefficient between Rudolph risk score and CAM-S maximum score. (B) Spearman correlation coefficient between SPPB and CAM-S maximum score. (C) Spearman correlation coefficient between adapted model risk score and CAM-S maximum score. Abbreviations: CAM, Confusion Assessment Methods; SPPB, Short Physical Performance Battery.

predictors that we considered for this model-SPPB components and established risk factors from the Rudolph model — were chosen a priori, which avoids overfitting from screening a wide range of predictors. Nonetheless, our model should be validated in an independent sample.

Our study has a few limitations. First, as our study was conducted in a single academic center treating predominantly white patients (180 out of 187 patients), the delirium incidence may be unique to our institution. While this limits generalizability of the absolute risk of delirium to other centers, we think that the relative increases remain applicable to other settings. Second, almost a quarter of screened patients were excluded due to refusal or logistical reasons (the research team was less available for assessment for patients whose procedure was scheduled urgently). These exclusions may have resulted in exclusion of sicker and frailer patients, leading to underestimation of the delirium incidence and the association of physical performance with delirium. Third, our modest sample size resulted in imprecise estimates of ORs for certain risk categories. Additionally, this impacted our ability to detect clinically meaningful differences in model discrimination. While the improvement in C-statistics by adding SPPB to the Rudolph model (TAVR 0.62 to 0.67 ) was statistically non-significant, it does not exclude the possibility of a clinically meaningful improvement in sensitivity 
(ie, at a specificity of 0.70 , sensitivity was 0.43 for the Rudolph model to 0.61 for the Rudolph model and SPPB combined). We acknowledge that our model showed moderate discrimination (C-statistic of 0.71), which is comparable to the performance of other clinical prediction models. ${ }^{40}$ Fourth, patients who were enrolled in the delirium sub-study tended to have lower surgical risk scores than patients enrolled prior to initiation of the delirium sub-study. This trend is consistent with the ongoing expansion of TAVR to lower-risk patients, ${ }^{41}$ and suggests that our model should be evaluated in a more contemporary population.

In conclusion, we found that physical performance is an independent risk factor for postoperative delirium after SAVR and TAVR. Our results support assessment of physical performance in addition to cognitive function to improve delirium risk prediction in patients being evaluated for aortic valve replacement.

\section{Key Points}

1. Poor physical performance, as measured using the Short Physical Performance Battery, is associated with higher risk of postoperative delirium.

2. Existing delirium prediction models do not generalize well to the unique setting of elective aortic valve replacement.

3. Delirium prediction improves when incorporating physical performance, depression, stroke, malnutrition, and cognition.

\section{Abbreviations}

CABG, Coronary Artery Bypass Grafting Surgery; CAM, Confusion Assessment Method; CI, Confidence Interval; MMSE, Mini-Mental State Examination; OR, Odds Ratio; SPPB, Short Physical Performance Battery; SAVR, Surgical Aortic Valve Replacement; TAVR, Transcatheter Aortic Valve Replacement.

\section{Disclosure}

Jeffrey J. Popma reports grants from Medtronic, Boston Scientific, and Abbott, and grants and personal fees from Edwards, outside the submitted work. Kimberly Guibone reports personal fees from Medtronic, outside the submitted work. Dae Hyun Kim reports grants from NIH, during the conduct of the study. Sandra M. Shi receives sponsorship from the Harvard Translational Research in Aging Training Program, T32 AG023480. The authors report no other potential conflicts of interest for this work.

\section{References}

1. Shi SM, Sung M, Afilalo J, et al. Delirium incidence and functional outcomes after transcatheter and surgical aortic valve replacement. J Am Geriatr Soc. 2019;1-9. doi:10.1111/jgs.15867.

2. Kim DH, Afilalo J, Shi SM, et al. Evaluation of changes in functional status in the year after aortic valve replacement. JAMA Intern Med. 2019;179(3):383-391. doi:10.1001/jamainternmed.2018.6738

3. Marcantonio ER, Solomon CG. Delirium in hospitalized older adults. N Engl J Med. 2017;377(15):1456-1466. doi:10.1056/NEJMcp1605501

4. Gottesman RF, Grega MA, Bailey MM, et al. Delirium after coronary artery bypass graft surgery and late mortality. Ann Neurol. 2010;67 (3):338-344. doi:10.1002/ana.21899

5. Saczynski JS, Marcantonio ER, Quach L, et al. Cognitive trajectories after postoperative delirium. $N$ Engl J Med. 2012;367(1):30-39. doi:10.1056/NEJMoa1112923

6. Hudetz JA, Iqbal Z, Gandhi SD, Patterson KM, Byrne AJ, Pagel PS. Postoperative delirium and short-term cognitive dysfunction occur more frequently in patients undergoing valve surgery with or without coronary artery bypass graft surgery compared with coronary artery bypass graft surgery alone: results of a pilot study. $J$ Cardiothorac Vasc Anesth. 2011;25(5):811-816. doi:10.1053/j.jvca.2010.05.003

7. Smith CR, Leon MB, Mack MJ, et al. Transcatheter versus surgical aortic-valve replacement in high-risk patients. $N$ Engl $J$ Med. 2011;364(23):2187-2198. doi:10.1056/NEJMoa1103510

8. Eide LSP, Ranhoff AH, Fridlund B, et al. Delirium as a predictor of physical and cognitive function in individuals aged 80 and older after transcatheter aortic valve implantation or surgical aortic valve replacement. J Am Geriatr Soc. 2016;64(6):1178-1186. doi:10.1111/ jgs. 14165

9. Carabello BA, Paulus WJ. Aortic stenosis. Lancet. 2009;373 (9667):956-966. doi:10.1016/S0140-6736(09)60211-7

10. Young J, Murthy L, Westby M, Akunne A, O'Mahony R; Guideline Development Group. Diagnosis, prevention, and management of delirium: summary of NICE guidance. BMJ. 2010;341:c3704. doi:10.1136/bmj.c3704.

11. Rudolph JL, Jones RN, Levkoff SE, et al. Derivation and validation of a preoperative prediction rule for delirium after cardiac surgery. Circulation. 2009;119(2):229-236. doi:10.1161/ CIRCULATIONAHA.108.795260

12. Bucerius J, Gummert JF, Borger MA, et al. Predictors of delirium after cardiac surgery delirium: effect of beating-heart (off-pump) surgery. J Thorac Cardiovasc Surg. 2004;127(1):57-64. doi:10.1016/S00225223(03)01281-9

13. Kazmierski J, Kowman M, Banach M, et al. Preoperative predictors of delirium after cardiac surgery: a preliminary study. Gen Hosp Psychiatry. 2006;28(6):536-538. doi:10.1016/j.genhosppsych.2006.08.007

14. Veliz-Reissmüller G, Torres HA, Van der Linden J, Lindblom D, Jönhagen ME. Pre-operative mild cognitive dysfunction predicts risk for post-operative delirium after elective cardiac surgery. Aging Clin Exp Res. 2007;19(3):172-177. doi:10.1007/BF03324686

15. Persico I, Cesari M, Morandi A, et al. Frailty and delirium in older adults: a systematic review and meta-analysis of the literature. $J \mathrm{Am}$ Geriatr Soc. 2018;66(10):2022-2030. doi:10.1111/jgs.15503

16. Laurie W, Shroyer A, Plomondon ME, Grover FL, Edwards FH. The 1996 coronary artery bypass risk model: the Society of Thoracic Surgeons adult cardiac national database. Ann Thorac Surg. 1999;67(4):1205-1208. doi:10.1016/S0003-4975(99)00206-4

17. Hoyl MT, Alessi CA, Harker JO, et al. Development and testing of a five-item version of the geriatric depression scale. $J$ Am Geriatr Soc. 1999;47(7):873-878. doi:10.1111/j.1532-5415.1999.tb03848.x

18. Guralnik JM, Simonsick EM, Ferrucci L, et al. A short physical performance battery assessing lower extremity function: association with self-reported disability and prediction of mortality and nursing home admission. J Gerontol. 1994;49(2):M85-M94. doi:10.1093/geronj/49.2.M85 
19. Pavasini R, Guralnik J, Brown JC, et al. Short physical performance battery and all-cause mortality: systematic review and meta-analysis. BMC Med. 2016;14(1):215. doi:10.1186/s12916-016-0763-7

20. Guralnik JM, Ferrucci L, Simonsick EM, Salive ME, Wallace RB. Lower-extremity function in persons over the age of 70 years as a predictor of subsequent disability. $N$ Engl J Med. 1995;332 (9):556-562. doi:10.1056/NEJM199503023320902

21. Albert MS, Levkoff SE, Reilly C, et al. The delirium symptom interview: an interview for the detection of delirium symptoms in hospitalized patients. Top Geriatr. 1992;5(1):14-21. doi:10.1177/ 002383099200500103

22. Inouye SK, Dyck CH, van Alessi CA, Balkin S, Siegal AP, Horwitz RI. Clarifying confusion: the confusion assessment method. Ann Intern Med. 1990;113(12):941. doi:10.7326/00034819-113-12-941

23. Inouye SK, Kosar CM, Tommet D, et al. The CAM-S: development and validation of a new scoring system for delirium severity in 2 cohorts. Ann Intern Med. 2014;160(8):526-533. doi:10.7326/M131927

24. Wong CL, Holroyd-Leduc J, Simel DL, Straus SE. Does this patient have delirium? JAMA. 2010;304(7):779. doi:10.1001/jama.2010.1182

25. Kim DH, Lee J, Kim CA, et al. Evaluation of algorithms to identify delirium in administrative claims and drug utilization database. Pharmacoepidemiol Drug Saf. 2017;26(8):945-953. doi:10.1002/ pds. 4226

26. Royston P, White I. Multiple imputation by chained equations (MICE): implementation in stata. J Stat Softw. 2011;45(4):1-20. doi:10.18637/jss.v045.i04

27. Sterne JAC, White IR, Carlin JB, et al. Multiple imputation for missing data in epidemiological and clinical research: potential and pitfalls. BMJ. 2009;339(7713):157-160. doi:10.1136/bmj.b2393

28. O'Brien KD. Pathogenesis of calcific aortic valve disease. Arterioscler Thromb Vasc Biol. 2006;26(8):1721-1728. doi:10.1161/ 01.ATV.0000227513.13697.ac

29. Afilalo J, Lauck S, Kim DH, et al. Frailty assessment in older adults undergoing transcatheter or surgical aortic valve replacement: the frailty-AVR study. J Am Coll Cardiol. 2016;67(13):8. doi:10.1016/ S0735-1097(16)30009-2

30. Assmann P, Kievit P, Van Der Wulp K, et al. Frailty is associated with delirium and mortality after transcatheter aortic valve implantation. Open Heart. 2016;3(2):e000478. doi:10.1136/openhrt-2016-000478
31. Jung P, Pereira MA, Hiebert B, et al. The impact of frailty on postoperative delirium in cardiac surgery patients. J Thorac Cardiovasc Surg. 2015;149(3):869-875.e2. doi:10.1016/J.JTCVS.2014.10.118

32. Leung JM, Tsai TL, Sands LP. Preoperative frailty in older surgical patients is associated with early postoperative delirium. Anesth Analg. 2011;112(5):1199-1201. doi:10.1213/ANE.0b013e31820c7c06

33. Partridge JSL, Fuller M, Harari D, Taylor PR, Martin FC, Dhesi JK. Frailty and poor functional status are common in arterial vascular surgical patients and affect postoperative outcomes. Int J Surg. 2015;18:57-63. doi:10.1016/J.IJSU.2015.04.037

34. Hausdorff JM, Yogev G, Springer S, Simon ES, Giladi N. Walking is more like catching than tapping: gait in the elderly as a complex cognitive task. Exp Brain Res. 2005;164(4):541-548. doi:10.1007/ s00221-005-2280-3

35. Sato T, Hatakeyama S, Okamoto T, et al. Slow gait speed and rapid renal function decline are risk factors for postoperative delirium after urological surgery. PLoS One. 2016;11(5):e0153961. doi:10.1371/ journal.pone.0153961

36. Yang FM, Inouye SK, Fearing MA, Kiely DK, Marcantonio ER, Jones RN. Participation in activity and risk for incident delirium. $J$ Am Geriatr Soc. 2008;56(8):1479-1484. doi:10.1111/j.15325415.2008.01792.x

37. Lee SS, Lo Y, Verghese J. Physical activity and risk of postoperative delirium. J Am Geriatr Soc. 2019;67(11):2260-2266. doi:10.1111/ jgs. 16083

38. Kuo H-K, Lipsitz LA. Cerebral white matter changes and geriatric syndromes: is there a link? J Gerontol a Biol Sci Med Sci. 2004;59 (8):M818-M826. doi:10.1093/gerona/59.8.M818

39. Arthur HM, Daniels C, McKelvie R, Hirsh J, Rush B. Effect of a preoperative intervention on preoperative and postoperative outcomes in low-risk patients awaiting elective coronary artery bypass graft surgery. Ann Intern Med. 2000;133(4):253. doi:10.7326/00034819-133-4-200008150-00007

40. D'Agostino RB, Grundy S, Sullivan LM, Wilson P. Validation of the Framingham coronary heart disease prediction scores: results of a multiple ethnic groups investigation. J Am Med Assoc. 2001;286 (2):180-187. doi:10.1001/jama.286.2.180

41. Grover FL, Vemulapalli S, Carroll JD, et al. 2016 annual report of the Society of Thoracic Surgeons/American College of Cardiology transcatheter valve therapy registry. $J$ Am Coll Cardiol. 2017;69 (10):1215-1230. doi:10.1016/j.jacc.2016.11.033
Clinical Interventions in Aging

\section{Publish your work in this journal}

Clinical Interventions in Aging is an international, peer-reviewed journal focusing on evidence-based reports on the value or lack thereof of treatments intended to prevent or delay the onset of maladaptive correlates of aging in human beings. This journal is indexed on PubMed Central, MedLine, CAS, Scopus and the Elsevier
Bibliographic databases. The manuscript management system is completely online and includes a very quick and fair peer-review system, which is all easy to use. Visit http://www.dovepress.com/ testimonials.php to read real quotes from published authors. 